\title{
Risk of chronic kidney disease in young adults with impaired glucose tolerance/ impaired fasting glucose: a retrospective cohort study using electronic primary care records
}

Ferozkhan Jadhakhan ${ }^{1 *}$, Tom Marshall ${ }^{1}$, Ronan Ryan ${ }^{1}$ and Paramjit Gill, ${ }^{1,2}$

\begin{abstract}
Background: The risk of chronic kidney disease (CKD) is known to be elevated in patients with diabetes mellitus but the risk of young adults aged 18 to 40 years with impaired glucose tolerance/impaired fasting glucose (IGT/IFG) developing CKD is not well characterised. Furthermore, progression of IGT/IFG to diabetes and subsequent CKD development is not well understood.

Methods: A retrospective cohort study was undertaken using The Health Improvement Network (THIN) database, a large dataset of electronic patient records. THIN database is jointly managed by IMS Health Real World Evidence Solution (http://www.epic-uk.org/index.html) and In Practice System (InPs). Cases were aged 18 to 40, with a diagnosis of IGT/IFG and registered at a practice contributing to THIN between 2000 and 2015. The study population consisted of 40,092 patients, including 21,454 (53.5\%) female and 18,638 (46.5\%) male. The median follow-up was approximately 2 years. The outcome was a diagnosis of CKD determined from either clinical coding or laboratory results. For the primary analysis the unadjusted and adjusted relative risk of CKD in IGT/IFG was compared to age, sex and practice matched controls with normoglycaemia. For the secondary analysis we compared the incidence of CKD before to after a diagnosis of type 2 diabetes (T2DM) in the IGT/IFG study cohort.
\end{abstract}

Results: The Incidence Rate Ratio (IRR) for CKD for IGT/IFG compared to normoglycaemia was 4.0 [95\% confidence interval (Cl), 3.2 to $5.1, P<0.001]$. The adjusted IRR was 2.6 [95\% Cl, 2.0 to 3.4, $P<0.001]$. The unadjusted IRR was 8.8 [95\% Cl, 7.7 to $10.0, P<0.001]$ after IGT/IFG patients had developed T2DM and the adjusted IRR was 6.3 [95\% Cl, 5.5 to $7.2, P<0.001]$.

Conclusion: Our results show that young IGT/IFG subjects are also at higher risk of developing CKD. This risk is modulated by the degree of baseline renal function and glucose tolerance, being higher in those developing T2DM.

Keywords: Impaired glucose tolerance, Impaired fasting glucose, Chronic kidney disease, The Health Improvement Network (THIN) database, Type 2 diabetes (T2DM), Read code, Incidence rate ratio (IRR)

\footnotetext{
*Correspondence: ferozkhan.jadhakhan@nhs.net; hiferozjad@gmail.com

${ }^{1}$ Primary Care Clinical Sciences, Institute of Applied Health Research,

University of Birmingham, Edgbaston, Birmingham B15 2TT, UK

Full list of author information is available at the end of the article
} 


\section{Background}

CKD is defined as a gradual loss of kidney function for more than three months with or without kidney damage [1]. It is common, progressive, usually asymptomatic [2] and can co-exist with other conditions [2]. The health burden of CKD is likely to rise because the prevalence of type 2 diabetes (T2DM) has been rapidly increasing worldwide [3]. Although the risk of CKD in patients with diabetes is relatively well established, the risk of young adults aged 18 to 40 years with IGT/IFG developing CKD is not well characterised. IGT/IFG is an intermediate state between normal glucose homeostasis and diabetic hyperglycaemia. Individuals with IGT/IFG have glucose levels higher than normal but not high enough for a diagnosis of diabetes [4]. In a ten year prospective study of 241 individuals with IGT, 15\% developed T2DM, 53\% reverted to normal glucose tolerance and $22 \%$ remained glucose intolerant [5]. Similarly, a 4 year prospective study conducted in 128 South African Indians with IGT at baseline, found that after 4 years, $50.4 \%$ progressed to diabetes, $24.8 \%$ persisted with IGT and 24.8 reverted to normoglycaemia [6]. There is some evidence that the incidence of CKD is elevated in individuals with IGT/IFG, but this is confined to specific populations [7-9]. Furthermore, the progression of IGT/IFG to T2DM and subsequent CKD development is not well understood. As many studies have used a single determination of glycaemic status at baseline, it is not clear whether the risk of developing a CKD event is confined to people with IGT/IFG who progress to overt diabetes or whether the risk is still increased among people with IGT/IFG even if they never develop diabetes. To address these considerations, we investigated the incidence of CKD in patients with IGT/IFG compared to matched controls with normoglycaemia. This was followed by a comparison of the incidence of CKD before and after a diagnosis of T2DM. The comparison was between the IGT/IFG period and the post T2DM period for all IGT/IFG patients who were in the study cohort.

\section{Methods}

\section{Study design}

This was a population based, retrospective, open cohort study using the electronic health record of patients registered with a family practice contributing to The Health Improvement Network (THIN) database.

\section{Data source}

THIN contains anonymised electronic patient records from over 600 UK general practices. As of September 2014 the database, held 13 million patients, of whom 3.6 million were actively registered with a general practice: more than 85 million person years or approximately $6 \%$ of the UK population [10] (http://www.epic-uk.org/our-data/ statistics.shtml). General practices contributing data to THIN use Vision patient records computer system which codes clinical data using the 5-byte Read code clinical classification [11]. Drug prescriptions issued can be linked to the British National Formulary [12]. Read code lists used to define the outcomes and conditions of interest can be found in Additional file 1. These lists were based on the Quality and Outcomes Framework (QOF) version (33.0) 2015 rulesets (http://www.hscic.gov.uk/qofbrv33).

\section{Study cohorts}

Individual practices were eligible to contribute patients from the later of one year after installation of Vision and their Acceptable Mortality Rate (AMR date) until the date of the last data collection (maximum February 2015). This ensured that they were using their records system fully and that the observation period for each patient was not overestimated [13]. Individual patients were eligible for inclusion in the study from the latest of the following four dates: practice eligibility date, patient registration date plus one year (to ensure patients did not have the outcome of interest (CKD) prior to entry), age 18 years, and the study start date (January 2000). Patients were followed up until the earliest of the following dates: age 41 years, transfer out of the practice or death, and their practice's last collection date.

\section{Cases (IGT/IFG definition)}

Cases with an incident diagnosis of IGT/IFG were identified using the earliest of the following events:

1. A Read coded diagnosis of IGT/IFG or Pre-diabetes - (Appendix 1)

2. Reported measurements of:

a. HbA1c in the range $42-47 \mathrm{mmol} / \mathrm{mol}[14,15]$

b. Fasting blood glucose (FPG) in the range 6.1$6.9 \mathrm{mmol} / \mathrm{L}[15,16]$

c. Oral Glucose Tolerance Test (OGTT) blood glucose in the range $\geq 7.8 \leq 11.1 \mathrm{mmol} / \mathrm{L}[15,16]$

The date of diagnosis of IGT/IFG became the index date.

\section{Exclusion criteria}

1. Patients with an index date prior to their cohort entry date

2. Patients with a diagnosis of diabetes (type 1 or type 2) prior to their index date

3. Patient cohort exit date was within 90 days of their index date

4. Patient diagnosed with diabetes (type 1 or type 2 ) within 30 days of their index date

5. Patients with a diagnosis of CKD prior to their index date 


\section{Matched controls}

Eligible IGT/IFG cases were matched to three normoglycaemic controls (without IGT/IFG or T2DM) on gender, age (within 2 years), and registration at the same family practice at the IGT/IFG case index date. Matched controls patients may not necessarily have a blood test to confirm their glycaemic status, suggesting that their glycaemic status may not be known but are generally assumed to be normoglycaemic. The same inclusion and exclusion criteria were applied to potential matches, except that they were required not to have a diagnosis of IGT/IFG on the index date of the IGT/IFG case.

\section{Follow-up}

IGT/IFG cases and matched patients were followed up until the earliest of the following dates: patient died; left practice; diagnosed with CKD; diagnosed with T2DM; last data collection from practice; or they reached the age of 41. For the secondary analysis IGT/IFG cases were not censored at T2DM diagnosis.

\section{Outcome (CKD definition)}

The outcome of interest was a diagnosis of incident CKD stage 1-5 identified using the earliest of the following events:

1. A clinically coded diagnosis of CKD stage (1-2/3-5) - (Appendix 1) or

2. A single estimated glomerular filtration rate (eGFR) of $\left(\geq 60 \mathrm{ml} / \mathrm{min} / 1.73 \mathrm{~m}^{2}\right.$ [CKD stage $\left.\left.1-2\right]\right)$ with additional evidence of kidney disease which include the following:

- A single albumin creatinine ratio (ACR) of ( $\geq 3 \mathrm{mg} / \mathrm{mmol}$ ) [17]

- A single protein creatinine ratio (PCR) of (50 mg/ mmol) [17]

- A single urinary albumin/protein loss of $0.5 \mathrm{~g} /$ day [17]

3. $\mathrm{eGFR}<60 \mathrm{ml} / \mathrm{min} / 1.73 \mathrm{~m} 2$ (CKD stage $3-5$ )

\section{Covariates}

Covariates (risk modifiers) were selected based on their biological plausibility and previously published epidemiological evidence $[18,19]$. These covariates were age, hypertension, heart failure (HF), atrial fibrillation (AF), cardiovascular disease (CVD) and exposure to a non-steroidal anti-inflammatory drug (NSAID). The latter was defined as a prescription during the year before the index date. All the other covariates were recorded at any time before the index date.

\section{Ethics approval}

THIN database (data collection process) obtained ethical approval from the South East Multi-centre Research
Ethics Committee (reference number: 07/H1102/103). The protocol for this study was reviewed and approved in June 2014 (study reference: 14-038) by the THIN Scientific Review Committee (SCR).

\section{Statistical analysis}

\section{- Descriptive statistics}

All statistical analyses were conducted using Stata version 13.1 (Stata Corp, College Station Texas, USA). Appropriate descriptive statistics were used to summarise the covariates and matching characteristics for those exposed and unexposed to IGT/IFG. Categorical variables were investigated using (Chi-square $\left[x^{2}\right]$ ) test and continuous variables were analysed using t-test. Since, length of follow-up had a highly skewed distribution Mann-Whitney U test was used to compare groups. For categorical variable (e.g., ethnicity) and continuous variables (e.g., BMI) a separate 'missing' category was created for those with missing data.

- Calculating incidence rate of CKD:

1. IGT/IFG compared to normoglycaemia

Adjusted and unadjusted IRRs for CKD in patients aged 18 to 40 years for the period January 2000 to February 2015 was estimated. Poisson regression was used to adjust for patient level covariates (age, hypertension, heart failure, atrial fibrillation, cardiovascular disease and steroid treatment).

\section{IGT/IFG period compared to post T2DM period}

Adjusted and unadjusted IRRs for CKD in patients aged 18 to 40 years for the period January 2000 to February 2015 was estimated. Poisson regression was used to adjust for patient level covariates.

\section{Results}

\section{Characteristics of matched cohort study population}

The study population consisted of 10,561 patients with IGT/IFG cases and 29,531 matched controls (Table 1). For both cases and controls median age at index date was 35 years, $54 \%$ were female and median follow-up was approximately 1.7 years. Compared to patients with normoglycaemia, patients with IGT/IFG were more likely to be of South Asian ethnicity, from areas of greater deprivation, and to have a BMI of $30.0 \mathrm{~kg} / \mathrm{m}^{2}$ or higher. Atrial fibrillation, heart failure and cardiovascular disease, hypertension and prescriptions for non-steroidal anti-inflammatory drugs were more frequent in IGT/IFG than normoglycaemic patients. During follow-up $11.9 \%$ of IGT/IFG and $0.4 \%$ of the normoglycaemia cohorts 
Table 1 Characteristics of the matched cohort (IGT/IFG compared to normoglycaemia)

\begin{tabular}{|c|c|c|c|c|c|}
\hline \multirow[b]{2}{*}{ Age, mean [SD] } & \multicolumn{2}{|c|}{$\begin{array}{l}\text { IGT/IFG } \\
{[N=10,561(26 \%)]}\end{array}$} & \multicolumn{2}{|c|}{$\begin{array}{l}\text { Normoglycaemia } \\
{[N=29,531(74 \%)]}\end{array}$} & \multirow{2}{*}{$\begin{array}{l}\text { P-Value } \\
0.015^{t+}\end{array}$} \\
\hline & 33.9 & {$[5.3]$} & 33.8 & {$[5.4]$} & \\
\hline Age, median [IQR] & 35 & {$[31,38]$} & 35 & {$[31,38]$} & \\
\hline Female, No. (\%) & 5659 & (53.6) & 15,795 & (53.5) & $0.863^{\dagger}$ \\
\hline Years of follow-up, mean [SD] & 2.3 & [2.1] & 2.3 & [2.0] & $0.684^{+++}$ \\
\hline Years of follow-up, median [IQR] & 1.68 & {$[0.81,3.16]$} & 1.67 & {$[0.79,3.14]$} & \\
\hline \multicolumn{6}{|l|}{ Ethnicity, No. (\%) } \\
\hline White & 3644 & (34.5) & 11,305 & (38.3) & $<0.001^{\dagger}$ \\
\hline Black & 392 & $(3.7)$ & 784 & $(2.7)$ & \\
\hline Asian & 1483 & $(14.0)$ & 1902 & $(6.4)$ & \\
\hline Chinese & 32 & $(0.3)$ & 102 & $(0.4)$ & \\
\hline Mixed & 63 & (0.6) & 219 & $(0.7)$ & \\
\hline Others & 130 & $(1.2)$ & 333 & $(1.1)$ & \\
\hline Unknown & 4817 & $(45.6)$ & 14,886 & $(50.5)$ & \\
\hline \multicolumn{6}{|l|}{ Deprivation quintile, No. (\%) } \\
\hline (least deprived) 1 & 1509 & $(14.3)$ & 5229 & $(17.7)$ & $<0.001^{+}$ \\
\hline 2 & 1575 & $(14.9)$ & 4985 & $(16.9)$ & \\
\hline 3 & 2177 & (20.6) & 6149 & (20.8) & \\
\hline 4 & 2463 & $(23.3)$ & 6319 & $(21.4)$ & \\
\hline (most deprived) 5 & 2232 & $(21.1)$ & 5020 & $(17.0)$ & \\
\hline Unknown & 605 & (5.7) & 1829 & $(6.2)$ & \\
\hline CVD at index date, No. (\%) & 190 & $(1.8)$ & 274 & $(0.9)$ & $<0.001^{+}$ \\
\hline HF at index date, No. (\%) & 29 & $(0.30)$ & 21 & $(0.07)$ & $<0.001^{\dagger}$ \\
\hline AF at index date, No. (\%) & 17 & $(0.16)$ & 21 & $(0.07)$ & $0.010^{\dagger}$ \\
\hline Hypertension at index date, No. (\%) & 1112 & $(10.5)$ & 548 & (1.9) & $<0.001^{\dagger}$ \\
\hline Prescription of NSAID, No. (\%) & 200 & (1.9) & 120 & $(0.4)$ & $<0.001^{\dagger}$ \\
\hline \multicolumn{6}{|l|}{ BMI $\left(\mathrm{kg} / \mathrm{m}^{2}\right)^{2}$, No. (\%) } \\
\hline$<20$ & 332 & (3.1) & 2191 & $(7.4)$ & $<0.001^{\dagger+}$ \\
\hline $20-24.9$ & 1542 & $(14.6)$ & 9452 & (32.0) & \\
\hline $25-29.9$ & 2420 & $(22.9)$ & 7028 & (23.8) & \\
\hline $30-34.9$ & 2104 & $(19.9)$ & 2868 & $(9.7)$ & \\
\hline $35-39.9$ & 1585 & $(15.0)$ & 1071 & (3.6) & \\
\hline$\geq 40$ & 1575 & $(15.0)$ & 644 & $(2.2)$ & \\
\hline Unknown & 1003 & (9.5) & 6277 & $(21.2)$ & \\
\hline BMI $\left(\mathrm{kg} / \mathrm{m}^{2}\right)$, mean $[\mathrm{SD}]$ & 26.0 & [5.7] & 32.1 & {$[8.2]$} & $<0.001^{+\dagger}$ \\
\hline BMI $\left(\mathrm{kg} / \mathrm{m}^{2}\right)$, median [IQR] & 31 & {$[26,37.1]$} & 24.9 & {$[22.1,28.7]$} & \\
\hline T2DM during follow-up, No. (\%) & 1262 & $(11.9)$ & 124 & $(0.4)$ & $<0.001^{\dagger}$ \\
\hline CKD during follow-up, No. (\%) & 182 & $(1.7)$ & 126 & $(0.4)$ & $<0.001^{\dagger}$ \\
\hline \multicolumn{6}{|l|}{ Country, No. (\%) } \\
\hline England & 8516 & (80.6) & 23,661 & $(80.0)$ & $0.688^{+}$ \\
\hline Northern Ireland & 270 & (2.6) & 791 & $(2.7)$ & \\
\hline Scotland & 804 & (7.6) & 2321 & (7.9) & \\
\hline Wales & 971 & (9.2) & 2758 & (9.3) & \\
\hline
\end{tabular}

Values are numbers (percentages) unless otherwise specified. $P$-values are from $X^{2}$ test $\left({ }^{\dagger}\right)$ for proportions or t-test $\left({ }^{+\dagger}\right)$ for continuous variables $($ age, $\mathrm{BMI}$ ) and Mann-Whitney $\mathrm{U}$ test $\left({ }^{+++}\right)$for follow-up

Abbreviations: CVD cardiovascular disease, $H F$ heart failure, $A F$ atrial fibrillation, NSAID non-steroidal anti-inflammatory drug, $B M I$ body mass index, $I Q R$ interquartile range 
developed T2DM. In addition 1.7\% of IGT/IFG cases were diagnosed with CKD compared to $0.4 \%$ in the normoglycaemia cohort.

Table 2 provides information about the number of CKD cases first recoded via Read code and first recorded via biomedical data in the IGT/IFG cohort.

\section{Incidence rate of CKD in persons aged 18 to 40 years with IGT/IFG compared to those with normoglycaemia} The unadjusted incidence of CKD per 100,000 personyears in persons with IGT/IFG was 4 times higher than individuals with normoglycaemia [IRR: 4.0; 95\% CI, 3.2 to 5.1, $P<0.001]$. After adjustment for age, sex, ethnic group, deprivation quintile, BMI categories, cardiovascular disease, heart failure, atrial fibrillation, hypertension and steroid use, incidence was reduced to approximately 3 times higher [IRR: 2.6; 95\% CI, 2.0 to 3.4, $P<0.001$ ] in persons with IGT/IFG (Table 3). In the adjusted analysis there was an increased incidence of CKD for each additional year of age at IGT/IFG diagnosis [IRR: $1.1 ; 95 \% \mathrm{CI}, 1.0$ to $1.1, P<0.001$ ] and an increased incidence in patients with a diagnosis of hypertension [IRR: 3.1; 95\% CI, 2.3 to $4.2, P<0.001$ ].

\section{Incidence rate of CKD in persons aged 18 to 40 years before and after T2DM}

The unadjusted incidence of CKD in patients with IGT/ IFG after T2DM diagnosis was approximately 9 times higher [IRR: 8.8; 95\% CI, 7.7 to $10.0, P<0.001$ ] compared to patients with IGT/IFG. After adjustment with potential confounders, the incidence of CKD remained high [IRR: 6.3; $95 \% \mathrm{CI}, 5.5$ to $7.2, P<0.001$ ] in patients who progressed to T2DM compared to those with IGT/IFG (Table 4). Of the adjusted covariates there was an increased incidence of CKD for each additional year of age at T2DM diagnosis [IRR: 1.3 (95\% CI, 1.2 to $1.3, P<0.001$ ] the incidence of CKD was also increased in patients with a diagnosis of hypertension [IRR: $1.6 ; 95 \% \mathrm{CI}, 1.4$ to $1.9, P<0.001$ ].

\section{Discussion}

The objective of this study was to investigate the incidence of CKD in a retrospective matched cohort analysis using the THIN database and found that young adults with IGT/ IFG were four times more at risk of developing CKD than individuals with normoglycaemia. After adjusting for age, sex, ethnic group, deprivation quintile, BMI categories, cardiovascular disease, heart failure, atrial fibrillation, hypertension and NSAID, the effect of CKD risk was attenuated

Table 2 Cases of CKD first recorded by Read code and first recorded by biomedical data in the IGT/IFG cohort

\begin{tabular}{llll}
\hline CKD results & Read code & Biomedical data & Total \\
\hline CKD cases recorded & 26 & 156 & 182 \\
\hline
\end{tabular}

Abbreviation: CKD chronic kidney disease
Table 3 Adjusted incidence rate of CKD in IGT/IFG compared to normoglycaemia

\begin{tabular}{|c|c|c|c|c|}
\hline Predictors & & IRR & $95 \%(\mathrm{Cl})$ & $P$-Value \\
\hline IGT/IFG vs normoglycaemia & & 2.6 & $(2.0,3.4)$ & $<0.001$ \\
\hline Female & & 1.1 & $(0.9,1.4)$ & 0.425 \\
\hline Age (in years) & & 1.1 & $(1.0,1.1)$ & $<0.001$ \\
\hline \multicolumn{5}{|l|}{ Ethnicity } \\
\hline White & Reference & 1.0 & & \\
\hline Asian & & 1.2 & $(0.8,1.7)$ & 0.389 \\
\hline Black & & 1.0 & $(0.6,1.9)$ & 0.955 \\
\hline Chinese & & 1.2 & $(0.2,8.7)$ & 0.845 \\
\hline Mixed & & 0.5 & $(0.1,3.3)$ & 0.439 \\
\hline Other & & 1.0 & $(0.4,2.8)$ & 0.970 \\
\hline Missing & & 0.7 & $(0.5,0.8)$ & 0.001 \\
\hline \multicolumn{5}{|l|}{ BMI $\left(\mathrm{kgs} / \mathrm{m}^{2}\right)$} \\
\hline$<20$ & Reference & 1.0 & & \\
\hline $20-24.9$ & & 1.9 & $(0.9,4.5)$ & 0.127 \\
\hline $25-29.9$ & & 2.5 & $(1.1,5.8)$ & 0.029 \\
\hline $30-34.9$ & & 3.2 & $(1.4,7.5)$ & 0.006 \\
\hline $35-39.9$ & & 2.6 & $(1.1,6.2)$ & 0.037 \\
\hline$\geq 40$ & & 3.2 & $(1.3,7.6)$ & 0.010 \\
\hline Missing & & 1.4 & $(0.6,3.5)$ & 0.452 \\
\hline \multicolumn{5}{|l|}{ Deprivation quintile } \\
\hline Least deprived 1 & Reference & 1.0 & & \\
\hline 2 & & 0.8 & $(0.5,1.3)$ & 0.394 \\
\hline 3 & & 1.1 & $(0.8,1.6)$ & 0.571 \\
\hline 4 & & 0.9 & $(0.6,1.4)$ & 0.756 \\
\hline Most deprived 5 & & 1.3 & $(0.9,1.9)$ & 0.123 \\
\hline Missing & & 1.4 & $(0.8,2.3)$ & 0.256 \\
\hline CVD & & 0.9 & $(0.4,2.3)$ & 0.905 \\
\hline HF & & 0.8 & $(0.1,6.2)$ & 0.872 \\
\hline AF & & 2.9 & $(0.4,21.0)$ & 0.285 \\
\hline Hypertension & & 3.1 & $(2.3,4.2)$ & $<0.001$ \\
\hline Steroid & & 1.1 & $(0.9,1.5)$ & 0.404 \\
\hline
\end{tabular}

Abbreviations: $B M I$ body mass index, $C V D$ cardiovascular disease, $H F$ heart failure, $A F$ atrial fibrillation, $\mathrm{Cl}$ confidence interval

but was still 2.6 times higher in individuals with IGT/IFG than those with normoglycaemia. Among the modifiable risk factors, hypertension was consistently linked to higher incidence of CKD. Individuals progressing from IGT/IFG to T2DM were approximately nine times more likely to be diagnosed with CKD following diabetes diagnosis than individuals with IGT/IFG. After adjustment for potential confounders, incidence of CKD was attenuated but still showed a significant association. The incidence was reduced to approximately 7 times higher in T2DM than the IGT/IFG cohort. 
Table 4 Adjusted incidence rate of CKD in T2DM compared to IGT/IFG

\begin{tabular}{|c|c|c|c|c|}
\hline Predictors & & IRR & $95 \%(\mathrm{Cl})$ & $P$-Value \\
\hline T2DM vs IGT/IFG & & 6.3 & $(5.5,7.2)$ & $<0.001$ \\
\hline Female & & 1.2 & $(1.0,1.4)$ & 0.008 \\
\hline Age (in years) & & 1.3 & $(1.2,1.3)$ & $<0.001$ \\
\hline \multicolumn{5}{|l|}{ Ethnicity } \\
\hline White & Reference & 1.0 & & \\
\hline Asian & & 0.8 & $(0.6,1.0)$ & 0.096 \\
\hline Black & & 0.8 & $(0.5,1.2)$ & 0.279 \\
\hline Chinese & & 0.6 & $(0.1,4.2)$ & 0.601 \\
\hline Mixed & & 0.9 & $(0.4,2.2)$ & 0.856 \\
\hline Other & & 0.8 & $(0.5,1.5)$ & 0.538 \\
\hline Missing & & 0.8 & $(0.7,0.9)$ & 0.001 \\
\hline \multicolumn{5}{|l|}{ BMI (kgs/m²) } \\
\hline$<20$ & Reference & 1.0 & & \\
\hline $20-24.9$ & & 2.1 & $(0.8,5.2)$ & 0.110 \\
\hline $25-29.9$ & & 2.6 & $(1.1,6.4)$ & 0.035 \\
\hline $30-34.9$ & & 3.2 & $(1.3,7.8)$ & 0.010 \\
\hline $35-39.9$ & & 3.3 & $(1.3,8.0)$ & 0.009 \\
\hline$\geq 40$ & & 2.8 & $(1.1,6.7)$ & 0.025 \\
\hline Missing & & 1.9 & $(0.7,4.8)$ & 0.181 \\
\hline \multicolumn{5}{|l|}{ Deprivation quintile } \\
\hline Least deprived 1 & Reference & 1.0 & & \\
\hline 2 & & 0.9 & $(0.7,1.1)$ & 0.390 \\
\hline 3 & & 1.0 & $(0.9,1.2)$ & 0.947 \\
\hline 4 & & 0.9 & $(0.7,1.1)$ & 0.349 \\
\hline Most deprived 5 & & 0.7 & $(0.6,0.9)$ & 0.008 \\
\hline Missing & & 1.0 & $(0.7,1.4)$ & 0.830 \\
\hline CVD & & 1.3 & $(0.9,2.0)$ & 0.231 \\
\hline HF & & 0.7 & $(0.2,2.2)$ & 0.514 \\
\hline AF & & 2.0 & $(0.7,5.4)$ & 0.174 \\
\hline Hypertension & & 1.6 & $(1.4,1.9)$ & $<0.001$ \\
\hline Steroid & & 1.0 & $(0.8,1.1)$ & 0.675 \\
\hline
\end{tabular}

Abbreviations: $B M I$ body mass index, $C V D$ cardiovascular disease, $H F$ heart failure, $A F$ atrial fibrillation, $\mathrm{Cl}$ confidence interval

\section{Strengths and limitations Strengths}

The strengths include the large sample size of the database (THIN contains medical records of over 12 million patients) which is approximately $6 \%$ of the UK population [10]. Generalisability was ensured by the fact that the dataset includes similar patients to those seen in primary care across the UK. This reflects the fact that data are collected in a noninterventional fashion during routine clinical practice.
Internal validity (reducing the effect of confounders) was ensured by matching patient's age, sex and general practice. Although the study was retrospective, data were collected prospectively, hence eliminating recall bias. Ascertainment of exposure and outcome of interest was carried out by both Read coded diagnosis and clinical measurements (e.g. HbA1c, ACR lab results). Additionally, the completeness of lab results has significantly improved since it is now directly fed into GP electronic patient records instead of entered manually. Therefore, improving ascertainment of IGT/IFG and CKD for this study and providing a reliable estimate of incident CKD in young adults with IGT/IFG. This occurred during the study period (2000-2015); earlier years may have had under reporting.

\section{Limitations}

The main limitation relates to the potential misclassification of patients with IGT/IFG (i.e. patients are identified as normoglycaemic when in fact they had IGT/IFG but were not identified because they had no blood tests). This would dilute the relationship between IGT/IFG and CKD because some of the controls have IGT/IFG and the likely association between IGT/IFG and risk of CKD may be reduced. Additionally, there is also potential for misclassification of patients with CKD (i.e. patients Read coded as CKD but with no laboratory reported evidence of reduced eGFR and patients with laboratory evidence of CKD but with no Read code labelling of CKD). However, for this study both laboratory evidence of reduced eGFR and Read code were used to identify incident CKD. There is incomplete data on some important confounding variables (e.g., ethnicity, BMI). Additionally, before automated reporting and recording, lab results were entered manually and in some cases only abnormal results may have been entered into the GP system: this could underestimate the incidence of CKD. There is also potential for ascertainment bias which may arise when patients who have frequent laboratory tests may be more likely to have both IGT/IFG and CKD diagnosed. Furthermore, the incidence and prevalence of recorded CKD depends on general practitioners (GPs) doing blood tests. This may underestimate the frequency of CKD and may also be biased because GPs are more likely to do blood tests for CKD in patients in whom they suspect CKD. This includes those with diabetes, hypertension, certain ethnic groups and those with IGT/ IFG. This is reflected in the number of CKD recorded via Read code and laboratory measurements.

\section{Comparison with other studies}

The results of this study appear to be consistent with previous studies showing an elevated risk of CKD in 
adults with IGT/IFG $[8,20,21]$. Watanabe and colleagues [9] in the Niigata Preventive Medicine Study, examining the risk of CKD in individual components of metabolic syndrome, found the incidence of IGT/IFG was approximately twice as high in subjects with IGT/ IFG as those with normoglycaemia. Furthermore, a retrospective study, using data from the Framingham Offspring cohort study examined the risk of CKD development in non-diabetic patients, found that patients with IGT/IFG were approximately two times more likely to develop CKD and those with diabetes were approximately four times more at risk of CKD [7]. Additionally, A prospective study, using data from the Italian Association of Clinical Diabetologists (Associazione Medici Diabetologi, AMD) initiative, examined risk factors associated with the development of CKD in patients aged ( $\geq 18$ years) with type 2 diabetes and without CKD or albuminuria at baseline. Patients were followed up between January 1, 2004 and June 30, 2008. During followup, patients who showed reduced kidney function and albuminuria were older males with poor glycaemic control, higher systolic blood pressure, hyperlipidaemia and on hypertensive and lipid lowering drugs [22]. The risk factors identified in this study are similar to those identified in the current study. The Framingham study contained data of 2398 patients aged ( $\geq 28$ years) and $47 \%$ were male compared to a study population of over 40,000 individuals aged 18 to 40 years in the current study. The gender distributions were similar in both studies. The follow-up period in the Yamagata and colleagues study was 10 years compared a shorter length of follow-up for individual patients in the current study (median 2 years approximately). In their study, Yamagata and colleagues included 113, 764 individuals aged ( $\geq 40$ years) and $33 \%$ were male compared to a study population of over 40,000 individuals aged 18 to 40 years and $46.4 \%$ male in the current study. These studies, however, did not report separate results in persons aged 18 to 40 years with IGT/IFG and risk of CKD, but rather results were presented for the population as a whole.

\section{Conclusion}

To the best of our knowledge, this study was the first population based study to address this question utilising data from a large generalisable cohort of general practices and their patients from across the UK. It used contemporary data, to investigate the incidence of CKD in young adults aged 18 to 40 years with IGT/IFG compared to those with normoglycaemia. This study provides evidence of an increased risk of CKD amongst young adults with IGT/IFG. Analyses revealed that patients progressing from IGT/IFG to T2DM are at an even higher risk of developing $\mathrm{CKD}$, providing further opportunity for individuals with IGT/IFG to consider lifestyle modification and have regular follow-up check of their kidney function to detect CKD at an early stage. Additionally, among the modifiable risk factors, hypertension was consistently linked to higher incidence of CKD. The result of this study shows the sizeable adverse effect of IGT/IFG on the development of CKD, and if left undetected may cause serious long term health issues and should be addressed at an early stage to reduce the future burden of CKD in patients with IGT/IFG.

\section{Additional file}

Additional file 1: Appendix. Read code lists. (DOCX 89 kb)

\section{Abbreviations}

ACR: Albumin creatinine ratio; AF: Atrial fibrillation; AMR: Acceptable mortality recording; BMI: Body Mass Index; BNF: British National Formulary; CHD: Coronary heart disease; $\mathrm{Cl}$ : Confidence interval; $\mathrm{CKD}$ : Chronic kidney disease;

CVD: Cardiovascular disease; EGFR: Estimated glomerular filtration rate; FPG: Fasting plasma glucose; HbA1c: Glycosylated haemoglobin; HF: Heart failure; HR: Hazards ratio; IFG: Impaired fasting glucose; IGR: Impaired glucose regulation; IGT: Impaired glucose tolerance; InPs: In Practice Systems; IQR: Interquartile range; IR: Incidence rate; IRR: Incidence Rate Ratio; NIHR: National Institute for Health and Care Excellence; NR: Not reported; NSAIDS: Non-steroidal anti-inflammatory drugs; OGTT: Oral Glucose Tolerance Test; PCR: Protein creatinine ratio; PYR: Person years; QOF: Quality and Outcomes Framework; RR: Relative risk; SCR: Serum Creatinine; SD: Standard deviation; T2DM: Type 2 Diabetes; THIN: The health improvement network database; UK: United Kingdom

\section{Acknowledgments}

This study forms part of FJ's PhD and is funded by the National Institute for Health Research School for Primary Care Research (NIHR SPCR) studentship grant. The views expressed are those of the author(s) and not necessarily those of the National Health Service, the National Institute for Health Research, the Department of Health or the University of Birmingham. Routinely collected electronic data used as part of this study was approved by a THIN Scientific Review Committee of the Cegedim Strategic Data Medical Research United Kingdom (the data provider).

\section{Funding}

This study forms part of FJ's PhD and was funded by the National Institute for Health Research (NIHR) School for Primary Care Research (SPCR). The views expressed in this publication are those of the authors and not necessarily those of the National Health Service, NIHR or the University of Birmingham. FJ as corresponding author had full access to the data and final responsibility for the decision to submit for publication.

\section{Availability of data and materials}

The data that support the findings of this study are available from [IMS Health Real World Evidence Solutions and In Practice System (InPS)] but restrictions apply to the availability of these data, which were used under license for the current study, and so are not publicly available. Data are however available from the authors upon reasonable request and with permission of [IMS Health Real World Evidence Solutions and In Practice System (InPs)]. THIN database is jointly managed by IMS Health Real World Evidence Solutions (http://www.epic-uk.org/ index.html) and In Practice System (InPs). THIN database obtained ethical approval for data collection from the South East Multicentre Research Ethics Committee (reference number: 07/H1102/103). Research undertaken using the database is therefore approved provided it has undergone independent scientific review. Researchers requiring access to THIN have to submit a study protocol justifying access and gain approval from one of THIN's Scientific Advisory Committees. Professor Tom Marshall (co-author) is a sub licence holder that covers full access to The Health Improvement Network (THIN) for the purpose of conducting large-scale epidemiological, clinical and health care utilisation studies. 


\section{Authors' contributions}

Contributions of the authors were as follows: FJ, PG and TM had the original research idea. FJ developed the research idea with contributions from PG and TM. RR carried out data extraction. RR provided statistical support analysing and interpreting the data. FJ drafted the manuscript, TP, PG and RR reviewed the manuscript and provided comments. All authors contributed to the interpretation of the data and revision of the manuscript. All authors read and approved the final manuscript.

\section{Ethics approval and consent to participate}

Routinely collected electronic data used as part of this study was approved by a THIN Scientific Review Committee of the Cegedim Strategic Data Medical Research United Kingdom (the data provider).

\section{Consent for publication}

The Health Improvement Network (THIN) is a large primary care database containing longitudinal anonymised medical records of patients. Access to these records is subject to approval from the respective scientific review board. Patients are given the option to have their de-personalised medical records collected for scientific research purposes, therefore patients consent are not required when working with anonymised patients records in the THIN database.

\section{Competing interests}

The author declares that they have no competing interests.

\section{Publisher's Note}

Springer Nature remains neutral with regard to jurisdictional claims in published maps and institutional affiliations.

\section{Author details}

${ }^{1}$ Primary Care Clinical Sciences, Institute of Applied Health Research, University of Birmingham, Edgbaston, Birmingham B15 2TT, UK. ${ }^{2}$ WMS Social Science and Systems in Health, University of Warwick, Coventry CV4 7AL, UK.

Received: 26 July 2017 Accepted: 25 January 2018

Published online: 26 February 2018

\section{References}

1. Stevens PE, Levin A. Evaluation and management of chronic kidney disease: synopsis of the kidney disease: improving global outcomes 2012 clinical practice guideline. Ann Intern Med. 2013;158:825-30.

2. National Institute for Health and Care Excellence (NICE). Quality standard 5 : chronic kidney disease, 2011. http://guidance.nice.org.uk. Accessed 15 Mar 2016.

3. Parmar MS. Chronic renal disease. BMJ. 2002:325:85-90.

4. Nathan DM, Davidson MB, DeFronzo RA, Heine RJ, Henry RR, Pratley R, et al. Impaired fasting glucose and impaired glucose tolerance: implications for care. Diabetes Care. 2007;30:753-9.

5. Keen H, Jarrett RJ, McCartney P. The ten-year follow-up of the Bedford survey (1962-1972): glucose tolerance and diabetes. Diabetologia. 1982;22:73-8.

6. Motala AA, Omar MA, Gouws E. High risk of progression to NIDDM in southAfrican Indians with impaired glucose tolerance. Diabelogia. 1993;42:556-63.

7. Fox CS, Larson MG, Leip EP, Meigs JB, Wilson PW, Levy D. Glycemic status and development of kidney disease: the Framingham heart study. Diabetes Care. 2005;28:2436-40.

8. Sun F, Tao Q, Zhan S. Metabolic syndrome and the development of chronic kidney disease among 118924 non-diabetic Taiwanese in a retrospective cohort. Nephrology (Carlton). 2010;15:84-92.

9. Watanabe H, Obata H, Watanabe T, Sasaki S, Nagai K, Aizawa Y. Metabolic syndrome and risk of development of chronic kidney disease: the Niigata preventive medicine study. Diabetes Metab Res Rev. 2010;26:26-32.

10. IMS Health Intelligence Applied. Statistics, 2015. http://csdmruk.cegedim. com/our-data/statistics.shtml. Accessed 18 Jan 2016.

11. UK Terminology Centre. Why migrate to SNOMED CT. London: NHS; 2011. Version 1.1

12. British National Formulary [BNF], 2014. http://www.bnf.org/bnf. Accessed 10 Feb 2016

13. Maguire A, Blak BT, Thompson M. The importance of defining periods of complete mortality reporting for research using automated data from primary care. Pharmacoepidemiol Drug Saf. 2009;18:76-83.
14. World Health Organisation. Guidelines Approved by the Review Committee. Use of Glycated Haemoglobin ( $\mathrm{HbA} 1 \mathrm{c}$ ) in the Diagnosis of Diabetes Mellitus. Abbreviated Report of a WHO Consultation. Geneva: World Health Organisation; 2011.

15. National Institute for Health and Care Excellence (NICE). Preventing Type 2 Diabetes: Risk Idebtification and Interventions for Individuals at High Risk (PH 38). 2012. http://www.nice.org.uk/guidance/ph38. Accessed 15 Mar 2016.

16. World Health Organisation (WHO). Definition and Diagnosis of Diabetes Mellitus and Intermediate Hyperglycaemia: Report of WHO/IDF consultation, 2006. http://www.idf.org/webdata/docs/WHO_IDF_definition_diagnosis_of_ diabetes.pdf. Accessed 26 Aug 2016.

17. National Institute for Health and Care Excellence (NICE). Chronic kidney disease in adults: assessment and management, 2014. http://www.nice.org. uk/guidance/cg182. Accessed 15 Mar 2016.

18. Hippisley-Cox J, Coupland C. Predicting the risk of chronic kidney disease in men and women in England and Wales: prospective derivation and externa validation of the QKidney scores. BMC Fam Pract. 2010;11:49.

19. Gansevoort RT, Correa-Rotter R, Hemmelgarn BR, Jafar TH, Heerspink HJ, Mann JF, et al. Chronic kidney disease and cardiovascular risk: epidemiology, mechanisms, and prevention. Lancet (Lond). 2013;382:339-52.

20. Tozawa M, Iseki C, Tokashiki K, Chinen S, Kohagura K, Kinjo K, et al. Metabolic syndrome and risk of developing chronic kidney disease in Japanese adults. Hypertens Res. 2007;30:937-43.

21. Yang T, Chu CH, Hsu CH, Hsieh PC, Chung TC, Bai CH, et al. Impact of metabolic syndrome on the incidence of chronic kidney disease: a Chinese cohort study. Nephrology (Carlton). 2012;17:532-8.

22. De Cosmo S, Viazzi F, Pacilli A, Giorda A, Gentile S, Russo G, et al. Predictors of chronic kidney disease in type 2 diabetes. Medicine. 2016;27:e4007.

\section{Submit your next manuscript to BioMed Central and we will help you at every step:}

- We accept pre-submission inquiries

- Our selector tool helps you to find the most relevant journal

- We provide round the clock customer support

- Convenient online submission

- Thorough peer review

- Inclusion in PubMed and all major indexing services

- Maximum visibility for your research

Submit your manuscript at www.biomedcentral.com/submit
) Biomed Central 